\title{
A UTILIZAÇÃO DOS PROTOCOLOS CLÍNICOS E DIRETRIZES TERAPÊUTICAS E A RACIONALIZAÇÃO DA JUDICIALIZAÇÃO DO DIREITO À SAÚDE
}

The utilization of Clinical Protocols and Therapeutic Guidelines and the rationalization of judicialization for the right to health

${ }^{1}$ Universidade Presbiteriana Mackenzie. São Paulo/SP, Brasil.

Correspondência: Paulo Vitor Bérgamo Braga. E-mail: paulovbbraga@gmail.com.

Recebido em: 19/09/2016. Aprovado: 01/12/2016. 


\section{RESUMO}

O artigo analisa a adoção dos Protocolos Clínicos e Diretrizes Terapêuticas (PCDTs) como parâmetro objetivo a ser utilizado pelos tribunais nas ações judiciais cujo objeto são prestações de natureza positiva voltadas à efetivação do direito à saúde. Tal referencial pode auxiliar o juiz na tarefa de decidir as demandas judiciais de fornecimento de medicamentos, garantindo a prevalência da racionalidade prevista pelo formulador das políticas públicas correlatas e, consequentemente, a utilização de argumentos técnico-políticos na distribuição de bens e recursos de saúde. Contudo, não se trata de um parâmetro absoluto, podendo o conteúdo do PCDT ser questionado, até mesmo judicialmente. Com isso, a ação judicial passa a ser utilizada como um instrumento de democratização e de controle social de escolhas públicas, o que pode vir a impactar a própria política de saúde.

\section{Palavras-Chave}

Direito à Saúde; Diretriz Terapêutica; Judicialização; Protocolo Clínico.

\section{ABSTRACT}

The article analyzes the adoption of the Clinical Protocols and Therapeutic Guidelines as an objective measure to be used by the courts in cases where the purpose is to offer positive benefits in terms of realization of the right to health. Such a benchmark can assist the judge in the task of deciding the legal situation of medication supplies, guaranteeing the prevalence of rationality as outlined in the formulation of related public policies and, consequently, the use of technical-political arguments in the distribution of healthcare goods and resources. However, this is not an absolute parameter, and the content of the CPTGs may be questioned, even judicially. With this, judicial action is used as an instrument of democratization and social control among public choices, which can impact health policy itself.

\section{Keywords}

Clinical Protocol; Judicialization; Right to Health; Therapeutic Guidelines. 


\section{EMENTA}

CONSTITUCIONAL, ADMINISTRATIVO E PROCESSUAL CIVIL. AGRAVO DE INSTRUMENTO. AÇÃO ORDINÁRIA. TUTELA ANTECIPADA. FORNECIMENTO DE MEDICAMENTO. NÃO INDICAÇÃO DO MINISTÉRIO DA SAÚDE PARA TRATAMENTO DA ENFERMIDADE QUE ACOMETE A AGRAVANTE. IMPOSSIBILIDADE. AUSÊNCIA DE PROVA INEQUÍVOCA DA VEROSSIMILHANÇA DAS ALEGAÇÕES. NECESSIDADE DE DILAÇÃO PROBATÓRIA. NÃO ATENDIMENTO AOS REQUISITOS DO ART. 273 DO CPC. AGTR IMPROVIDO. 1. A decisão agravada, proferida na ação ordinária de origem, indeferiu o pedido de tutela antecipada, por considerar que existem diversas manifestações técnicas sobre a inaptidão do medicamento solicitado pela requerente para a moléstia que lhe acomete, ressaltando inclusive seus efeitos adversos, explicitando os motivos que levaram a não recomendação da utilização do medicamento pelo protocolo do SUS para o tratamento de melanoma disseminado. 2. As alegações apresentadas pela agravante não lograram infirmar a fundamentação da decisão agravada, no sentido de que o medicamento solicitado (Ipilimumab) é não recomendado pelo Ministério da Saúde para pacientes com melanoma disseminado, que parece ser o caso da ora agravante. 3. Demonstrada a não indicação do medicamento para a enfermidade que acomete a agravante, e não tendo a mesma apresentado qualquer argumento contrário a tal contraindicação, não se verifica a verossimilhança de suas alegações, impedindo a concessão da antecipação de tutela pretendida. 4. Se não restou comprovada a indicação do medicamento solicitado para a enfermidade que acomete a agravante, sendo necessária ampla dilação probatória, há que se indeferir o pedido de tutela antecipada. 5. Não cabe ao Poder Judiciário decidir acerca da administração ou não de determinado medicamento ao paciente, sendo tal decisão de caráter eminentemente médico, mas também não incumbe ao Estado-juiz determinar aos entes estatais que forneçam um certo medicamento ao paciente quando o Ministério da Saúde contraindica a sua administração. 6. Agravo de instrumento improvido. (PROCESSO: 08002575420164050000, AG/SE, DESEMBARGADOR FEDERAL MANOEL ERHARDT, $1^{\text {a }}$. TURMA, JULGAMENTO: 14/03/2016).

\section{Introdução}

O fenômeno da judicialização dos direitos sociais vem, há quase duas décadas, exigindo que os operadores do direito, os atores sociais e a própria Administração Pública adaptem o modelo clássico de solução de conflitos de direitos intersubjetivos, gerando uma evidente ampliação dos debates atinentes a sua implementação.

A Constituição de 1988 (CF/88) previu expressamente a importância da participação da sociedade na construção da política de saúde ${ }^{1}$, permitindo a abertura

\footnotetext{
${ }^{1}$ Podemos citar, como exemplos, o artigo 194, parágrafo único, inciso VII, que elenca o caráter democrático e descentralizado da administração dos subsistemas de seguridade social, bem como o artigo 198, inciso III, que estabelece a participação da comunidade como uma das diretrizes do Sistema Único de Saúde. BRASIL. Constituição da República Federativa do Brasil de 1988. Disponível em: <http://www.planalto.gov. br/ccivil_03/constituicao/constituicaocompilado.htm>. Acesso em: 31 maio 2107.
} 
do sistema e formando o substrato legal da chamada "participação da sociedade na produção do Direito Sanitário"2. Contudo, o conceito de política pública - entendida como programa de ação governamental constituído por uma série de atos e processos que visam à consecução de objetivos previamente definidos, unidos por uma finalidade comum ${ }^{3}$ - exige um planejamento e uma racionalidade que nem sempre a judicialização oferece.

Essa dualidade, evidenciada após a promulgação da $\mathrm{CF} / 88$, momento em que houve uma ampliação do rol de direitos sociais até então previstos nos textos constitucionais, trouxe demandas de caráter prestacional para a apreciação do Judiciário, transformando esse Poder em um importante locus de disputas de natureza distributiva. A ação judicial transformou-se em um canal de participação popular, capaz de gerar a correção ou readequação dos rumos de políticas concretas. Essa situação, no entanto, trouxe uma série de dúvidas quanto aos limites de atuação da esfera judicial dentro do ciclo de formação e implementação das políticas públicas.

Além do problema dos limites da atuação do Judiciário, há grandes divergências quanto à amplitude da proteção do direito à saúde na $\mathrm{CF} / 88$, principalmente no tocante ao conteúdo imediatamente exigível da expressão "atendimento integral". Como consequência, as ações judiciais, por vezes, acabam interferindo no planejamento realizado pelos administradores públicos e impactando, na prática, a garantia do direito à saúde da coletividade.

Dessa forma, faz-se necessário o estabelecimento de parâmetros objetivos para a solução de conflitos que envolvam a efetivação de políticas públicas, muitas vezes resultantes de falhas ou insuficiências nas próprias políticas. Com isso, a atuação dos operadores do direito e de setores da sociedade civil voltada à exigibilidade, individual ou coletiva, do direito à saúde não é negada e, de outro lado, fixam-se parâmetros concretos para a tomada de decisões administrativas ${ }^{4}$.

\section{A utilização dos protocolos clínicos como parâmetro objetivo na judicialização do fornecimento de medicamentos}

Embora ainda de forma incipiente, é possível constatar que cresce no Judiciário a preocupação em observar parâmetros claros e objetivos na concessão judicial

\footnotetext{
${ }^{2}$ Essa expressão foi cunhada por Fernando Aith. AITH, Fernando. Curso de direito sanitário: a proteção do direito à saúde no Brasil. 1. ed. São Paulo: Quartier Latin, 2007. p. 155.

${ }^{3} \mathrm{BUCCl}$, Maria Paula Dallari. O conceito de política pública em direito. In: BUCCI, Maria Paula Dallari (Org.). Políticas públicas: reflexões sobre o conceito jurídico. 1. ed. São Paulo: Saraiva, 2006. p. 39.

${ }^{4}$ No tocante às particularidades da judicialização da saúde e à tentativa de criar parâmetros objetivos, indicamos interessante artigo de Sueli Gandolfi Dallari, que condensa toda a problemática. DALLARI, Sueli. Aspectos particulares da chamada judicialização da saúde. Revista de Direito Sanitário, Brasil, v. 14, n. 1, p. 77-81, jun. 2013. Disponível em: <http://www.revistas.usp.br/rdisan/article/view/56624>. Acesso em: 31 maio 2017. http://dx.doi.org/10.11606/issn.2316-9044.v14i1p77-81.
} 
de medicamentos ${ }^{5}$. É exatamente nesse contexto que podemos situar o Agravo de Instrumento $08002575420164050000 / S E$, julgado pela $1^{\text {a }}$ Turma do Tribunal Regional Federal da $5^{\text {a }}$ Região, de relatoria do desembargador federal Manoel Erhardt ${ }^{6}$.

Referido recurso foi interposto contra decisão de primeiro grau que indeferiu pedido de tutela antecipada para concessão do medicamento ipilimumab, em razão de o mesmo ter sido contraindicado para o tratamento de melanoma disseminado (a doença do autor da demanda).

O desembargador federal Manoel Erhardt manteve a decisão agravada, deixando expressamente consignado na ementa do julgamento que:

5. Não cabe ao Poder Judiciário decidir acerca da administração ou não de determinado medicamento ao paciente, sendo tal decisão de caráter eminentemente médico, mas também não incumbe ao Estado-juiz determinar aos entes estatais que forneçam um certo medicamento ao paciente quando o Ministério da Saúde contraindica a sua administração ${ }^{7}$.

Como se pode constatar, a referida decisão aplica como parâmetro objetivo a impossibilidade de fornecimento de medicamento expressamente contraindicado para determinada doença pelas autoridades competentes, reforçando a importância, na proteção do direito à saúde, das decisões técnicas dos órgãos regularmente constituídos para esse fim.

A Lei n. 8.080/1990 ${ }^{8}$, alterada pela Lei n. $12.401 / 2011^{9}$, estabelece a observância dos Protocolos Clínicos e Diretrizes Terapêuticas (PCDTs) como um dos parâmetros objetivos para o fornecimento de medicamentos pelo Sistema Único de Saúde (SUS) - conforme redação do artigo 19-M, inciso I, que restringe o conteúdo da chamada "assistência terapêutica integral".

No âmbito da incorporação, exclusão ou alteração de novas tecnologias no SUS, bem como no tocante à criação e alteração dos PCDTs, o artigo 19-Q da Lei n. 8.080/1990

\footnotetext{
${ }^{5} \mathrm{O}$ ministro Gilmar Mendes, no Agravo Regimental na Suspensão de Tutela Antecipada 175/CE, após a Audiência Pública n. 4, estabeleceu uma série de parâmetros objetivos para a concessão de medicamentos, cujo voto merece leitura. SUPREMO TRIBUNAL FEDERAL. Agravo regimental na suspensão de tutela antecipada 175/CE. Rel. Min. Gilmar Mendes. Tribunal Pleno, data do julgamento: 17/03/2010.

${ }^{6}$ TRIBUNAL REGIONAL FEDERAL DA 5 ${ }^{\mathrm{a}}$ REGIÃO. Agravo de Instrumento 08002575420164050000/SE, Relator Desembargador Federal Manoel Erhardt, $1^{\text {a }}$ Turma, data do julgamento: 14/03/2016.

${ }^{7}$ Id. Ibid.

${ }^{8}$ BRASIL. Lei Federal n. 8.080, de 19 de setembro de 1990. Dispõe sobre as condições para a promoção, proteção e recuperação da saúde, a organização e o funcionamento dos serviços correspondentes e dá outras providências. Disponível em: <http://www.planalto.gov.br/ccivil_03/leis/L8080.htm>. Acesso em: 31 maio 2017.

${ }^{9}$ BRASIL. Lei Federal n. 12.401, de 28 de abril de 2011. Altera a Lei no 8.080, de 19 de setembro de 1990, para dispor sobre a assistência terapêutica e a incorporação de tecnologia em saúde no âmbito do Sistema Único de Saúde - SUS. Disponível em: <http://www.planalto.gov.br/ccivil_03/_ato2011-2014/2011/lei/ I12401.htm>. Acesso em: 31 maio 2017.
} 
estabelece ser incumbência da Comissão Nacional de Incorporação de Tecnologias no SUS (Conitec) auxiliar tecnicamente o Ministério da Saúde na tomada de suas decisões.

A atuação da Comissão se baseia em duas premissas básicas, previstas expressamente no parágrafo $2^{\circ}$ do citado artigo 19-Q: de um lado, a análise científica baseada em evidências, buscando determinar a eficácia, acurácia, efetividade e segurança do medicamento, produto ou procedimento (inciso I); e, de outro, a avalição da relação entre o custo e a efetividade da tecnologia, tendo como paradigma os bens já incorporados ao sistema público de saúde (inciso II).

No caso do melanoma disseminado, a proibição da utilização do medicamento pleiteado em juízo consta dos PCDTs em oncologia, tendo como fundamento a ausência de evidências concretas do "benefício global em termos de sobrevida"10.

E é exatamente nesse ponto que reside o principal aspecto da adoção de tal parâmetro: a vedação à utilização do medicamento fundamentada no benefício global em termos de sobrevida, que leva em consideração não apenas a eficácia do medicamento, mas também seu custo-efetividade.

Em um dos estudos utilizados pela Conitec para justificar a não ministração do ipilimumab, os pesquisadores notaram que a sobrevida global dos pacientes que utilizaram o referido medicamento em conjunto com dacarbazina (recomendado pelos protocolos clínicos) foi de 11,2 meses, enquanto que a sobrevida global dos pacientes que receberam dacarbazina em conjunto com placebo foi de 9,1 meses $^{11}$.

Além disso, segundo a Nota Técnica n. 236/2013 do Núcleo de Avaliação de Tecnologias em Saúde, solicitada em outro processo, a diferença entre o custo de ambos os medicamentos é gritante: enquanto o tratamento com dacarbazina custaria R $\$ 5.518,80$, o custo do fornecimento do ipilimumab, no caso específico avaliado pela citada nota técnica, seria de $\mathrm{R} \$ 254.402,76^{12}$.

A utilização de argumentos econômicos em sentido lato (incluída a relação custo-efetividade) para justificar o não fornecimento de medicamentos não costuma ser aceita pelos tribunais, apesar de serem considerados pelo administrador na formulação da política pública ${ }^{13}$.

\footnotetext{
${ }^{10}$ BRASIL. Ministério da Saúde. Secretaria de Atenção à Saúde. Protocolos clínicos e diretrizes terapêuticas em Oncologia. Brasília-DF: Ministério da Saúde, 2014. p. 221.

${ }^{11}$ ROBERT, Caroline; THOMAS, Luc, BONDARENKO, Igor et al. Ipilimumab plus Dacarbazine for Previously Untreated Metastatic Melanoma. The New England Journal of Medicine, v. 326, n. 26, p. 2520, 2011. Disponível em: <http://www.nejm.org/doi/pdf/10.1056/NEJMoa1104621>. Acesso em: 05 set. 2016.

${ }^{12}$ NÚCLEO DE AVALIAÇÃO DE TECNOLOGIAS EM SAÚDE. Nota Técnica 236/2013. Disponível em: <http:// www.cnj.jus.br/files/conteudo/destaques/arquivo/2015/04/7013f6a5289dce21248b15af6931b 120. pdf>. Acesso em: 04 set. 2016.

${ }^{13} \mathrm{O}$ fato de argumentos econômicos (por exemplo, o custo de determinado medicamento) fazerem parte do processo de formulação de uma política pública não significa que eles tenham prioridade frente a outros argumentos, como no caso das doenças raras ou das negligenciadas, sobre as quais a obrigação de atuação do Estado é evidente.
} 
De toda forma, é certo que a utilização dos protocolos clínicos como parâmetro para a judicialização não retira a possibilidade de o magistrado, de acordo com as provas presentes nos autos e diante das especificidades do caso concreto, concluir pela necessidade de fornecer medicamento não previsto na política pública ${ }^{14},{ }^{15}$. Note-se que, na própria ementa examinada, o desembargador relator deixa consignado que a parte não apresentou argumentos contrários à contraindicação do medicamento, inclusive quanto a eventuais efeitos adversos, o que confirma a possibilidade de produção probatória nesse sentido.

Isso demonstra que as diretrizes contidas nos protocolos clínicos não são absolutas; antes disso, permitem iluminar o ponto controvertido relativo aos fatos da demanda e servem como parâmetro para a solução do litígio - ainda que este possa trazer situações não previstas nos instrumentos adotados pela política de saúde, mas que devem ser levadas em consideração.

\section{Alguns impactos da observância dos PCDTs como parâmetro objetivo}

A utilização dos protocolos clínicos como parâmetro objetivo que orienta as decisões em matéria de políticas públicas de saúde abre a possibilidade de questionamento judicial de seus conteúdos, tornando os próprios critérios utilizados para a determinação das vedações e prescrições neles previstas sujeitos a um maior controle social.

A interpretação ampliativa do conteúdo do direito à saúde - segundo a qual o Estado deve fornecer qualquer medicamento pleiteado judicialmente, ainda que em confronto à política pública estabelecida - acaba por relativizar o papel da Relação Nacional de Medicamentos Essenciais (Rename) e dos PCDTs. Tais programas são fruto de escolhas políticas por meio das quais são estabelecidas metas concretas - de acordo com os meios e recursos postos à disposição do poder público - para atingir, progressivamente, o objetivo da universalização do acesso a medicamentos. Nesse sentido, imprimem racionalidade às políticas públicas de saúde, ainda que isso não signifique que seu conteúdo não possa ser questionado.

\footnotetext{
${ }^{14}$ Nesse sentido, citamos o Agravo de Instrumento 08073709320154050000/SE, de relatoria do próprio desembargador federal Manoel Erhardt, no qual ele fornece medicamento não previsto na política pública de saúde em razão da não eficácia, no caso concreto, de cinco esquemas diferentes de quimioterapia. TRIBUNAL REGIONAL FEDERAL DA 5 ${ }^{a}$ REGIÃO. Agravo de Instrumento 08073709320154050000/SE, Relator Desembargador Federal Manoel Erhardt, $1^{\text {a }}$ Turma, data do julgamento: 20/02/2016.

${ }^{15}$ Segundo o Enunciado 61 da II Jornada de Direito da Saúde do Conselho Nacional de Justiça, que altera o Enunciado 4 da I Jornada: “Os Protocolos Clínicos e Diretrizes Terapêuticas (PCDT) são elementos organizadores da prestação farmacêutica, de insumos e de procedimentos, e não limitadores. Assim, no caso concreto, quando todas as alternativas terapêuticas previstas no respectivo PCDT já tiverem sido esgotadas ou forem inviáveis ao quadro clínico do paciente usuário do SUS, pelo princípio do art. 198, II da CF, pode ser determinado judicialmente o fornecimento, pelo Sistema Único de Saúde, do fármaco, insumo ou procedimento não protocolizado". CONSELHO NACIONAL DE JUSTIÇA. II Jornada de direito da saúde. Disponível em: <http://www.cnj.jus.br/files/conteudo/destaques/ arquivo/2015/05/96b5b10aec7e595 4fcc1978473e4cd80.pdf>. Acesso em: 31 maio 2017.
} 
Se não há consenso quanto aos limites de atuação do Judiciário no controle de políticas públicas, o fato é que existem parâmetros constitucionais objetivos ${ }^{16}$, além dos critérios consubstanciados nos protocolos, que devem ser respeitados pelo administrador e pelo legislador, e que servem como vetores para a interpretação das demandas concretas trazidas ao Judiciário nesse campo. É o caso dos objetivos fundamentais de redução das desigualdades sociais e regionais previstos no artigo $3^{\circ} \mathrm{da} \mathrm{CF} / 88$; dos princípios administrativos explícitos (artigo 37); da prioridade de ações de saúde preventiva e da necessária participação da sociedade no Sistema Único de Saúde (artigos 198, II e III) ${ }^{17}$; entre outros.

Nesse ponto, o Ministério Público e a Defensoria Pública, que já se tornaram importantes atores na garantia dos interesses difusos e coletivos relacionados à saúde - especialmente de grupos vulneráveis, tais como idosos, crianças, adolescentes e indígenas -, adquirem uma função ainda mais relevante na garantia do direito à saúde, controlando as próprias escolhas públicas, principalmente a partir dos instrumentos de tutela coletiva.

Abordando a possibilidade de atuação do Ministério Público na judicialização de políticas públicas, afirma Hugo Nigro Mazzilli que

A ação civil pública ainda se presta para que o Ministério Público possa questionar políticas públicas, quando do exercício de suas funções no zelo para que os Poderes Públicos e os serviços de relevância pública observem os direitos assegurados na Constituição. Com certeza, não poderá o Ministério Público pedir ao Poder Judiciário que administre no lugar do administrador; contudo, poderá cobrar em juízo a aplicação de princípios da Administração que possam estar sendo descurados, e, com isso, restaurar a legalidade. Também não poderá o Ministério Público estar movido por critérios político-partidários; entretanto, sua ação tem inegável caráter político, no sentido técnico da expressão, ou seja, a instituição ministerial pode legitimamente questionar atos de governo, que, entre outras hipóteses, firam o princípio da legalidade, configurem desvio ou abuso de poder, ou divirjam dos princípios da moralidade, eficiência ou razoabilidade, entre outros que devem informar a Administração ${ }^{18}$.

Esse modo de controle, aliás, possibilita corrigir uma das principais críticas à judicialização individual da saúde: a alegação de que o fornecimento de

\footnotetext{
${ }^{16}$ DUARTE, Clarice Seixas. O duplo regime jurídico do direito à saúde na CF/88: direito fundamental de caráter social e direito público subjetivo. Pensar: revista de ciências jurídicas. Fortaleza, v. 17, n. 2, p. 437, 2012. Disponível em: <http://ojs.unifor.br/index.php/rpen/article/view/2311>. Acesso em: 05 set. 2016. ${ }^{17}$ BRASIL. Constituição da República Federativa do Brasil de 1988, cit.

${ }^{18}$ MAZZILLI, Hugo Nigro. A defesa dos interesses difusos em juízo: meio ambiente, consumidor, patrimônio cultural, patrimônio público e outros interesses. 26. ed. rev., ampl. e atual. São Paulo: Saraiva, 2013. p. 141.
} 
tecnologias não ofertadas pelo SUS àqueles que recorrem ao Judiciário geraria uma situação de iniquidade em relação àqueles que se submetem às escolhas feitas pelo administrador/legislador via políticas públicas. A partir do momento em que a própria escolha pública é contestada judicialmente no âmbito coletivo, a decisão trará uma resposta aplicável em larga escala, potencializando os eventuais efeitos positivos da demanda ao estendê-los a todos os que se encontrem em uma situação semelhante (ou seja, necessitando da prestação objeto de discussão judicial).

Por outro lado, outra consequência da adoção dos PCDTs como parâmetro objetivo é o aprofundamento do debate sobre o uso de recursos públicos pelo Estado, a relação custo-efetividade das tecnologias e a adequação das escolhas do administrador, evitando aquilo que Canotilho denomina de "metodologia fuzzy", isto é, que não adentra os complexos fatores que condicionam o direito que está sendo concretizado ${ }^{19}$.

Como alertado pelo Relatório Mundial da Saúde de 2010, da Organização Mundial da Saúde (OMS), "os fundos comuns nunca serão capazes de cobrir 100\% da população para $100 \%$ dos custos e $100 \%$ dos serviços necessários. Os países ainda terão de fazer escolhas difíceis quanto à melhor utilização desses fundos"20.

Com a adoção dos PCDTs como parâmetro - tal como na decisão analisada - e com as escolhas políticas aclaradas por meios de justificativas técnicas baseadas em evidência, há, ao mesmo tempo, uma maior segurança do administrador, que terá a possibilidade de planejar a política de saúde, e dos atores sociais, que terão um referencial técnico objetivo estabelecido, passível de questionamento (até mesmo judicial) cujos resultados poderão impactar a (re)formulação da própria política pública de saúde.

\section{Referências}

AITH, Fernando. Curso de direito sanitário: a proteção do direito à saúde no Brasil. 1. ed. São Paulo: Quartier Latin, 2007.

BRASIL. Ministério da Saúde. Secretaria de Atenção à Saúde. Protocolos clínicos e diretrizes terapêuticas em Oncologia. Brasília-DF: Ministério da Saúde, 2014.

BUCCI, Maria Paula Dallari. O conceito de política pública em direito. In: BUCCI, Maria Paula Dallari (Org.). Políticas públicas: reflexões sobre o conceito jurídico. 1. ed. São Paulo: Saraiva, 2006.

\footnotetext{
${ }^{19}$ CANOTILHO, José Joaquim Gomes. Estudos sobre direitos fundamentais. 1. ed. Coimbra: Coimbra Ed., 2004. p. 100-101.

${ }^{20}$ ORGANIZAÇÃO MUNDIAL DA SAÚDE. Relatório Mundial da Saúde 2010: financiamento dos sistemas de saúde: o caminho para a cobertura universal, 2010. p. 2. Disponível em: <http://www.who.int/whr/2010/ whr10_pt.pdf>. Acesso em: 05 set. 2016.
} 
CANOTILHO, José Joaquim Gomes. Estudos sobre direitos fundamentais. 1. ed. Coimbra: Coimbra Ed., 2004.

DALLARI, Sueli. Aspectos particulares da chamada judicialização da saúde. Revista de Direito Sanitário, Brasil, v. 14, n. 1, p. 77-81, jun. 2013. Disponível em: <http://www. revistas.usp.br/rdisan/article/view/56624>. Acesso em: 31 maio 2017. http://dx.doi. org/10.11606/issn.2316-9044.v14i1p77-81.

DUARTE, Clarice Seixas. O duplo regime jurídico do direito à saúde na CF/88: direito fundamental de caráter social e direito público subjetivo. Pensar: revista de ciências jurídicas. Fortaleza, v. 17, n. 2, 2012. Disponível em: <http://ojs.unifor.br/index.php/rpen/ article/view/2311>. Acesso em 05 set. 2016.

MAZZILLI, Hugo Nigro. A defesa dos interesses difusos em juízo: meio ambiente, consumidor, patrimônio cultural, patrimônio público e outros interesses. 26. ed. rev., ampl. e atual. São Paulo: Saraiva, 2013.

ORGANIZAÇÃO MUNDIAL DA SAÚDE. Relatório Mundial da Saúde 2010: financiamento dos sistemas de saúde: o caminho para a cobertura universal, 2010. Disponível em: <http://www.who.int/whr/2010/whr10_pt.pdf >. Acesso em: 05 set. 2016.

NÚCLEO DE AVALIAÇÃO DE TECNOLOGIAS EM SAÚDE. Nota Técnica 236/2013. Disponível em: <http://www.cnj.jus.br/files/conteudo/destaques/arquivo /2015/04/7013f6a5289dce21248b15af6931b120.pdf>. Acesso em: 04 set. 2016.

ROBERT, Caroline; THOMAS, Luc, BONDARENKO, Igor et al. Ipilimumab plus Dacarbazine for Previously Untreated Metastatic Melanoma. The New England Journal of Medicine, v. 326, n. 26, 2011. Disponível em: <http://www.nejm.org/doi/pdf/ 10.1056/ NEJMoa1104621>. Acesso em 05 set. 2016.

Clarice Seixas Duarte - Doutora em Filosofia e Teoria Geral do Direito pela Universidade de São Paulo. Professora do Programa de Pós-Graduação Stricto Sensu em Direito Político e Econômico da Universidade Presbiteriana Mackenzie. São Paulo/SP, Brasil.

Paulo Vitor Bérgamo Braga - Mestre em Direito Político e Econômico pela Universidade Presbiteriana Mackenzie. Pesquisador dos Grupos de Pesquisa "Direito Sociais e Políticas Públicas" e "O Sistema de Seguridade Social", ambos vinculados à Universidade Presbiteriana Mackenzie. São Paulo/SP, Brasil. E-mail: paulovbbraga@gmail.com. 